\title{
Arthroscopic Cartilage Regeneration Facilitating Procedure: A Decompressing Arthroplasty for Knee Osteoarthritis
}

\section{Shaw-Ruey Lyu ( $\nabla$ srlyu@seed.net.tw )}

Dalin Tzu Chi General Hospital https://orcid.org/0000-0003-3797-4848

Jung-pin Hung

Dalin Tzu Chi Hospital

Chia-Chen Hsu

Dalin Tzu Chi Hospital

\section{Yu-Ruei Chen}

Dalin Tzu Chi Hospital

Chih-Wen Lin

Dalin Tzu Chi Hospital

\section{Technical advance}

Keywords: osteoarthritis; medial plica; medial abrasion phenomenon; medial abrasion syndrome; decompression; arthroplasty; arthroscopy; cartilage regeneration

Posted Date: June 25th, 2020

DOI: https://doi.org/10.21203/rs.3.rs-32928/v1

License: (1) (1) This work is licensed under a Creative Commons Attribution 4.0 International License.

Read Full License 


\section{Abstract}

Background The effectiveness of arthroscopic treatment for knee osteoarthritis (OA) has been subject to debate. This study presents an innovative concept of arthroscopic management for knee OA and investigates its clinical outcome.

Methods An arthroscopic cartilage regeneration facilitating procedure (ACRFP) to eliminate medial abrasion phenomenon and decompress the patello-femoral joints was performed on 693 knees of 411 patients with knee OA, mean age 60 years (34-90). The Knee Society score (KSS) and the knee injury and osteoarthritis outcome score (KOOS) were used for subjective outcome study. Roentgenographic changes for all cases and magnetic resonance imaging (MRI) variations for twenty randomly selected cases were evaluated for objective outcomes.

Results There were 634 knees in 369 patients (93.7\%) available with more than 3 years of follow-ups (mean 40 months, SD 9). The overall subjective satisfactory rate was $91.1 \%$. The KSS and all subscales of the KOOS improved statistically. Reversal of the degeneration process of cartilage was observed in $80.1 \%$ of the whole series (radiographic outcome) and in $72.2 \%$ of the 18 randomly selected cases (oneyear MRI outcome study). Gender and OA severity were related to subjective outcomes. Age, body mass index (BMI), pre-operative hyaluronic acid injection, OA severity, and the type and severity of the medial plica are important predictors of radiographic outcomes. An analysis of failed cases reaffirmed the need for early ACRFP and skillful post-operative care.

Conclusion ACRFP is an effective treatment for knee OA. If performed in time, it can satisfy most patients and might modify their degeneration process.

\section{Background}

Knee OA remains a tremendous public health concern worldwide, both in terms of health-related quality of life and financial burden of disease [1], [2]. Since the initiating events that result in cartilage degradation are poorly understood, the literature appears to be inconsistent and inconclusive in demonstrating disease modification in the early process of chondral degradation by modalities such as injections of platelet-rich plasma (PRP) [3], mesenchymal stem cell [4], or chondral debridement and transplantation of the degenerated cartilage by cultured chondrocytes, periosteum or perichondrium [5].

Since 2006, a series of studies [6]-[12] regarding medial abrasion phenomenon (MAP) as a cause of knee OA have been conducted. These studies put forward the role of MAP in the pathogenesis of knee OA [13]. Consequently, a technique of arthroscopic medial release (AMR) [14] and the concept of arthroscopic cartilage regeneration facilitating procedure (ACRFP) [15] were developed for the treatment of knee OA. The outcomes of these series and the subsequent positive feedback from clinical application of this concept as a knee health promotion option (KHPO) for the treatment of knee OA [16] persuaded us to further standardize the procedure of this technique and scrutinize its clinical outcomes. We propose that 
ACRFP offers these benefits: the eradication of damaging MAP and decompression, which should be maintained by skillful postoperative care to provide damaged cartilage an opportunity for regeneration.

\section{Methods}

\section{The patients}

In a one-year period, 411 consecutive patients (693 knees) who received the ACRFP for knee OA were prospectively followed as part of an Institutional Review Board Registry. There were 99 (24.4\%) males and $312(75.6 \%)$ females with a mean age at the time of surgery of 60 (34-90). The medial compartment was mainly involved in 638 knees (92.1\%) of 363 patients and the lateral compartment was mainly involved in 55 knees $(7.9 \%)$ of 48 patients. The clinical staging of the knee $\mathrm{OA}$ by both physical and rentgenographic examinations described in Table 1 was given by the senior author. Patients with OA of any stage and who have been treated conservatively for more than six months were included. The duration of symptoms, history of having previous operation, glucosamine sulfate intake or receiving hyaluronic acid (HA) injections for more than one year were recorded. Patients were excluded if they had instability due to previous ligament injury or OA due to malunion of fracture. Twenty bilateral stage III female patients older than 60 with 40 knees were randomly selected for magnetic resonance imaging (MRI) studies.

Table 1. Clinical staging for OA of individual knee compartments based on rentgenographic and clinical findings.

\begin{tabular}{ccccc}
\hline & \multicolumn{2}{c}{ Rentgenographic findings } & \multicolumn{2}{c}{ Clinical findings } \\
Stage & Joint space narrowing & Osteophyte & Deformity & Stability \\
\hline I & Doubtful & No & Normal & Stable \\
II & Definite, no more than 1/2 & Doubtful & $<5^{\circ}$ & Stable \\
\hline III & Marked, more than 1/2 & Definite & $5-10^{\circ}$ & Stable \\
\hline IV & Completely obliterated & Marked & $>10^{\circ}$ & Stable \\
\hline V & Completely obliterated & Marked & $>10^{\circ}$ & Unstable \\
\hline
\end{tabular}

\section{Radiographic Protocol}

The radiographic protocol described in previous publication[15] was followed in this study. To assess the tibiofemoral joint space, the standing extended anteroposterior radiographs of the knee were obtained at $55 \mathrm{kVp}$ with a focus of

$1.25 \mathrm{~mm}^{2}$ and a focus-to-film distance of $100 \mathrm{~cm}$ with the patella in central position. Merchant's view was used to assess the patellofemoral joint space. It was obtained at 45 degrees of knee flexion with X-ray beam projected caudad at 30 degrees. For magnification correction in each examination, a 4.0-mm steel ball was affixed to the skin over the head of the fibula (standing extended AP view) and center of patella (Merchant's view) to permit correlation of the joint space width measurements.

\section{MRI protocol}

MR images for bilateral knees of all selected subjects were obtained using 1.5T scanner (GE signa HDxt) and lower extremity coils, before and one year after ACRFP. The following sequences were acquired and used for image analysis: axial T2 weighted fast spin echo (FSE) with fat 
saturation (FS) sequence (TR/TE: 4967/81 ms, matrix: 256x224, slice thickness/interval: 3/3, NEX:2); axial intermediate weighted FSE sequence (TR/TE: 1000/41 ms, matrix: 256x224, slice thickness/interval: 3/3, NEX:1); sagittal T2 weighted FSE FS sequence (TR/TE: 4250/80 ms, matrix: 256x192, slice thickness/interval: 3/3, NEX:1); sagittal intermediate weighted FSE sequence (TR/TE: 1000/41 ms, matrix: 256x224, slice thickness/interval: 3/3, NEX:1); coronal intermediate weighted FSE sequence (TR/TE: 1000/41 ms, matrix: 256x224, slice thickness/interval: 3/3, NEX:1).

\section{Surgical procedure (Video 1)}

Either spinal or general anesthesia was used for this procedure. Throughout the procedure, patients were put in a supine position and bloodless surgical field was obtained by pneumatic tourniquet. A 4-millimeter 30-degree arthroscope with a 6.5-millimeter sheath was used. An electric shaver and hand instruments were used as necessary.

\section{Arthroscopic examination}

The arthroscopic examination was performed through the inferolateral portal. The presence of medial plica related MAP and its sequelae (Fig 1) was first investigated. The type and grading of the medial plica and the grading of two cartilaginous lesions (foci A and B) on the medial femoral condyle described in the previous publication [6] were recorded. The degree of cartilage degradation in each compartment was verified according to Outerbridge classification [17]. Whether the menisci were torn or not is also noted.

\section{Medial release}

Medial release was performed as described in previous publication [14]. The adequacy of the release was checked by passing the tip of the scope under the patella and verified if the previously tight medial patellofemoral joint space (Fig 2a) could be easily passed through and the medial retinaculum visualized (Fig 2b).

\section{Percutaneous lateral release}

After the medial release, the patella would always deviate laterally (Fig 2c). Lateral release was performed by inserting a No.11 scalpel into the inferolateral portal and cutting the lateral retinaculum percutaneously. The extent and adequacy of the release could be evaluated by direct vision through arthroscope (Fig 2d).

\section{Synovectomy and chondroplasty}

Any focal synovitis or loose chondral flaps on the cartilaginous surface was removed as conventional arthroscopic debridement for knee OA. No bony procedure such as drilling or microfracture was performed. Thorough irrigation was performed to remove debris in the knee joint at the end of the procedure.

\section{Post-operative management}


From the day of surgery, full range of motion, full weight bearing and free ambulation were allowed as tolerated. A suction drain was used for 24-48 hours. The patient was discharged 2 days after the operation. Home exercise programs, including quadriceps strengthening and passive range of motion, were emphasized. The compliance of patients regarding the home exercise program was strictly monitored by case managers. No supplementary treatment including oral glucosamine sulfate, steroid injections or intraarticular injection of HA or PRP was given throughout the post-operative follow-up period.

\section{Follow-up and evaluation of clinical outcome}

Patients returned for monthly evaluation for three months after surgery. Thereafter, they were examined during their annual follow-up visits. Both pre- and post-operative KSS and KOOS were compared for subjective outcome evaluation. Patients were asked by our case managers to answer direct questions about their satisfaction with ACRFP using a categorical scale prepared for our studies[14], [15]: excellent (free of symptoms, no limitation in activities), good (greatly improved, occasional pain, normal activities), fair (same as pre-operative condition, no improvement), and poor (has received or considered further operative treatment). The outcome was regarded as satisfactory if subjective satisfaction was "excellent" or "good". The inquiry questioning about patients' subjective satisfaction and the evaluation of KSS and KOOS were conducted by case managers. All investigations focused on individual knees in bilateral cases.

\section{Evaluation of x-ray outcome}

The pre-operative standing extended anteroposterior and Merchant's views were compared with those taken at the last clinic follow-up on a picture archiving communication system (PACS) workstation (INFINITT Healthcare Co., Ltd.). The interpreting parameters included femoral-tibial angle (FTA), minimum joint space width (MJSW), and the surface contour of the joint lines. The interpretation resulted in a judgement that the knee was better, the same, or worse following the ACRFP operation.

\section{MRI analysis}

Baseline and follow-up MR images of knee were transferred to a PACS workstation and reviewed. The presence and grade of cartilage, meniscal, and bone marrow lesions (bone marrow edema and cysts) were assessed using a modified whole organ MRI score (WORMS) [18], [19]. The sum and maximum scores (WORMS Sum and WORMS max) were recorded for each category, and difference of scores between baseline and follow-up images ( $\triangle$ WORMS Sum) were calculated.

All radiographic and MR images were analyzed by two radiologists (LCW with 15 years, CYR with 6 years in musculoskeletal imaging) independently. In any mismatched cases, a consensus was achieved by coreading.

\section{Statistical analysis}


Statistical analysis was carried out using JMP (Version 5.0.1.2, SAS Institute Inc., Cary, NC, USA). All values were presented with means and standard deviations. An one-way analysis of variance (ANOVA) to detect differences in the distribution of patient age in each stage of osteoarthritis was used to make comparisons. Analyses for comparing preoperative and postoperative KSS, KOOS, femoral-tibial angle, and joint space width were performed using the paired $t$ test. A two-sided $p$-value $(p<0.05)$ was considered statistically significant. Rogistic regression was used to analyze the response of subjective and radiographic outcomes as a function of age, BMI, duration of symptom, pre-operative femorotibial angle (FTA) and joint space width (JSW). Pearson's chi-square or Fisher's exact test were used for the analyses of the response of subjective and radiographic outcomes as a function of categorical variables (gender, glucosamine sulfate intake, HA injection, previous operation, meniscus tear, preoperative staging, type and severity of medial plica, and grading of cartilage damage over hidden lesions and each compartment).

\section{Results}

There were 634 knees in 369 patients available for thorough outcome study including x-ray examinations, KSS and KOOS evaluations. Sixteen of 196 knees (8.2\%) with grade II OA in nine patients, twenty-six of 414 knees $(6.3 \%)$ with grade III OA in seventeen patients, and two of 83 knees $(2.4 \%)$ with grade IV OA in two patients did not return to complete the follow-up. The total follow-up rate was $93.7 \%$ (including 14 unsatisfied patients who preferred to go to other hospitals for arthroplasty after the first annual check-up) and the mean follow-up period was 40 months (SD 9). The mean age and distribution of different stage of OA stratified by the main involved compartment were shown in Table 2. Medial compartment was mainly involved in 611 knees (94.1\%) of 357 patients and lateral compartment was mainly involved in 38 knees (5.9\%) of 26 patients. Eighteen patients (90\%) with 36 knees of the 20 randomly selected patients returned for the one-year follow-up MRI evaluation.

Table 2. Age distribution of different stage of OA stratified by main involved compartment

\begin{tabular}{cccc}
\hline & Medial Compartment & Lateral Compartment & Total \\
\hline Stage & Age (SD) / No. & Age (SD) / No. & Age (SD) / No. \\
\hline II & $64^{*}(10) / 178$ & $61^{*}(11) / 2$ & $64^{*}(10) / 180$ \\
III & $70(9) / 355$ & $68(8) / 33$ & $70(9) / 388$ \\
IV & $70(9) / 78$ & $71(17) / 3$ & $70(9) / 81$ \\
\hline Total & $68(10) / 611$ & $68(9) / 38$ & $60(10) / 649$ \\
\hline
\end{tabular}

* Statistically significant compared to that of stage III and IV by comparisons for each pair using paired $\mathrm{t}$ test $(\mathrm{p}<0.05)$

Subjective outcome assessment (Table 3) was satisfactory in 591 knees (91.1\%). Those with stage II had higher satisfaction rate $(p=0.008)$. The Knee Society scores and all subscales of KOOS were statistically improved in both medial and lateral compartment groups (Tables 4 and 5). The FTA improved from 0.45 (SD 4.33) to 0.59 (SD 4.45) ( $p=0.0397)$ in the medial compartment group and didn't show statistical 
difference in the lateral compartment group (9.39/SD 4.10 to 9.66/SD 4.48; $p=0.5113$ ). The JSW increased from 2.73 (SD 1.34) to 2.98 (SD 1.43) ( $p<0.0001$ ) in the medial compartment group and from 3.19 (SD 1.09) to 3.66 (SD 1.19) ( $p=0.0013)$ in the lateral compartment group. The radiographic outcome revealed overall reversal of the degeneration process in $80.1 \%$ of the whole series (Table 6). For the whole series and the medial compartment group, the rate of reversal of the degeneration process was higher in stage II knees $(p=0.001)$. There was no statistical difference in the rate of reversal of the degeneration process between the medial compartment group (80.0\%) and the lateral compartment group (89.5\%) $(p=0.106)$. The MRI outcomes (Table 7) showed reversal of the degeneration process in $72.2 \%$ of cartilage, $63.9 \%$ of bone marrow edema, $63.9 \%$ of bone cyst and $91.7 \%$ of meniscus categories of WORMS.

Table 3. Subjective Outcomes of different stage of OA stratified by main involved compartment

\begin{tabular}{|c|c|c|c|c|c|c|c|c|c|c|c|}
\hline \multirow[t]{2}{*}{ Stage } & \multicolumn{5}{|c|}{ Medial compartment $(\mathrm{N}=611)$} & \multicolumn{5}{|c|}{ Lateral compartment $(\mathrm{N}=38)$} & Tot \\
\hline & E† & G & $\mathrm{F}$ & $\mathrm{P}$ & Sat. (\%) & $\mathrm{E}$ & G & $\mathrm{F}$ & $\mathrm{P}$ & Sat. (\%) & \\
\hline II & $\begin{array}{r}74.2 \\
(132)\end{array}$ & $\begin{array}{l}23.0 \\
(41)\end{array}$ & $\begin{array}{l}2.8 \\
(5)\end{array}$ & 0 & 97.2 & $\begin{array}{l}50.0 \\
(1)\end{array}$ & 0 & $50.0(1)$ & 0 & 50.0 & $96.7 *$ \\
\hline III & $\begin{array}{c}56.3 \\
(200)\end{array}$ & $\begin{array}{c}32.1 \\
(114)\end{array}$ & $\begin{array}{c}5.1 \\
(18)\end{array}$ & $\begin{array}{c}6.5 \\
(23)\end{array}$ & 88.4 & $\begin{array}{l}57.6 \\
(19)\end{array}$ & $\begin{array}{l}30.3 \\
(10)\end{array}$ & $\begin{array}{l}9.1 \\
(3)\end{array}$ & $\begin{array}{l}3.0 \\
(1)\end{array}$ & 87.9 & 88.4 \\
\hline IV & $\begin{array}{l}47.4 \\
(37) \\
\end{array}$ & $\begin{array}{r}43.6 \\
(34) \\
\end{array}$ & $\begin{array}{l}1.3 \\
(1) \\
\end{array}$ & $\begin{array}{l}7.7 \\
(6) \\
\end{array}$ & 91.0 & $\begin{array}{c}33.3 \\
(1) \\
\end{array}$ & $\begin{array}{c}66.7 \\
(2) \\
\end{array}$ & 0 & 0 & 100.0 & 91.4 \\
\hline Total & $\begin{array}{c}60.4 \\
(369)\end{array}$ & $\begin{array}{c}30.9 \\
(189)\end{array}$ & $\begin{array}{c}3.9 \\
(24)\end{array}$ & $\begin{array}{c}4.8 \\
(29)\end{array}$ & 91.0 & $\begin{array}{l}55.3 \\
(21)\end{array}$ & $\begin{array}{l}31.6 \\
(12)\end{array}$ & $\begin{array}{c}10.5 \\
(4)\end{array}$ & $\begin{array}{l}2.6 \\
(1)\end{array}$ & 86.9 & 91.1 \\
\hline
\end{tabular}

† E: excellent; G: good; F: fair; P: poor; Sat.: satisfied = E + G, presented as \% (N)

* Statistically significant by comparisons for each pair using paired t test $(\mathrm{p}<0.05)$

Table 4. Pre-operative and post-operative Knee Society score for different stage of OA stratified by main involved compartment 


\begin{tabular}{|c|c|c|c|c|c|c|c|c|}
\hline \multirow[t]{2}{*}{ Stage } & \multicolumn{2}{|c|}{ Pain (SD) } & \multicolumn{2}{|c|}{ Function (SD) } & \multicolumn{2}{|c|}{ Pain (SD) } & \multicolumn{2}{|l|}{$\begin{array}{l}\text { Function } \\
\text { (SD) }\end{array}$} \\
\hline & Pre-op. & Post-op. & Pre-op. & Post-op. & Pre-op. & Post-op. & Pre-op. & Post-op. \\
\hline II & $\begin{array}{c}68.8 \\
(13.0)\end{array}$ & $\begin{array}{l}89.8 \\
(9.8)\end{array}$ & $\begin{array}{c}58.6 \\
(16.1)\end{array}$ & $\begin{array}{c}86.2 \\
(14.3)\end{array}$ & $\begin{array}{c}74.5 \\
(20.5)\end{array}$ & $\begin{array}{c}87.5 \\
(10.6)\end{array}$ & $40.0(28.3)$ & $\begin{array}{c}55.0 \\
(21.2)\end{array}$ \\
\hline III & $\begin{array}{c}65.8 \\
(12.9)\end{array}$ & $\begin{array}{c}86.0 \\
(11.0)\end{array}$ & $\begin{array}{c}53.5 \\
(16.7)\end{array}$ & $\begin{array}{c}79.2 \\
(17.1)\end{array}$ & $\begin{array}{c}65.9 \\
(13.0)\end{array}$ & $\begin{array}{c}84.6 \\
(10.5)\end{array}$ & $57.7(16.1)$ & $\begin{array}{c}74.5 \\
(18.0)\end{array}$ \\
\hline IV & $\begin{array}{c}62.4 \\
(14.6)\end{array}$ & $\begin{array}{c}80.5 \\
(15.0)\end{array}$ & $\begin{array}{c}47.3 \\
(16.9)\end{array}$ & $\begin{array}{c}74.8 \\
(17.4)\end{array}$ & $\begin{array}{l}68.0 \\
(6.1)\end{array}$ & $\begin{array}{l}80.3 \\
(9.5)\end{array}$ & $40.0(20.0)$ & $\begin{array}{c}60.0 \\
(40.0)\end{array}$ \\
\hline Total & $\begin{array}{c}66.3 \\
(13.1)\end{array}$ & $\begin{array}{c}86.4 \\
(11.7)\end{array}$ & $\begin{array}{c}54.2 \\
(16.9)\end{array}$ & $\begin{array}{c}80.7 \\
(16.8)\end{array}$ & $\begin{array}{c}66.5 \\
(12.7)\end{array}$ & $\begin{array}{c}84.4 \\
(10.3)\end{array}$ & $55.4(17.5)$ & $\begin{array}{c}72.4 \\
(20.3)\end{array}$ \\
\hline $\begin{array}{c}\mathrm{p} \\
\text { value }\end{array}$ & \multicolumn{2}{|c|}{$<0.0001$} & \multicolumn{2}{|c|}{$<0.0001$} & \multicolumn{3}{|c|}{$<0.0001$} & \\
\hline
\end{tabular}

Table 5. Pre-operative and post-operative KOOS of different stage of OA stratified by main involved compartment

\begin{tabular}{|c|c|c|c|c|c|c|c|c|c|c|c|}
\hline & & & & & & & & & & & \\
\hline Stage & Jumber) & Pre-op. & Post- & Pre- & Post- & Pre- & Post- & Pre- & Post- & Pre- & Post- \\
\hline & & & & & & & & & & & \\
\hline II & Med. & 66.8 & 88.4 & 52.9 & 84.2 & 71.1 & 91.5 & 40.6 & 68.2 & 43.4 & 70.2 \\
\hline & (178) & (17.0) & (14.0) & (15.2) & $(14.0)$ & (19.0) & (11.7) & $(26.4)$ & $(27.4)$ & $(20.1)$ & (22.6) \\
\hline & Lat. & 58.5 & 90.3 & 32.0 & 78.6 & 67.5 & 81.6 & 27.5 & 47.3 & 47.0 & 58.3 \\
\hline & $(2)$ & (7.8) & (13.7) & (15.6) & (10.1) & (2.1) & (3.1) & (17.7) & (10.6) & $(4.2)$ & (11.3) \\
\hline III & Med. & 63.1 & 86.0 & 52.9 & 81.1 & 68.1 & 86.6 & 30.1 & 55.4 & 38.7 & 66.4 \\
\hline & (355) & (16.1) & (15.0) & (15.8) & (16.2) & (16.6) & (15.2) & (20.4) & (32.2) & (17.4) & $(25.1)$ \\
\hline & Lat. & 59.8 & 83.2 & 45.6 & 73.8 & 63.3 & 83.9 & 29.5 & 44.1 & 38.1 & 61.0 \\
\hline & (33) & (18.1) & (12.6) & (19.1) & (15.0) & (22.8) & (11.8) & $(26.0)$ & (33.4) & (19.4) & (22.7) \\
\hline IV & Med. & 62.0 & 84.3 & 51.9 & 78.2 & 66.1 & 85.3 & 27.7 & 49.6 & 39.5 & 63.3 \\
\hline & (78) & (17.7) & (16.5) & (14.8) & (18.5) & (18.1) & (15.5) & (21.7) & (31.5) & $(20.0)$ & $(27.4)$ \\
\hline & Lat. & 60.3 & 77.0 & 46.3 & 66.6 & 57.7 & 75.4 & 20.0 & 45.0 & 37.7 & 62.5 \\
\hline & (3) & (5.9) & $(20.6)$ & $(12.9)$ & $(31.3)$ & $(21.6)$ & $(21.5)$ & $(13.2)$ & $(26.5)$ & $(11.0)$ & $(37.5)$ \\
\hline Total & Med. & 64.1 & 86.5 & 52.8 & 81.6 & 68.7 & 87.9 & 32.8 & 58.4 & 40.2 & 67.1 \\
\hline & (611) & (16.7) & (14.9) & (15.5) & $(16.0)$ & (17.6) & (14.5) & (23.0) & (31.5) & (18.6) & $(24.8)$ \\
\hline & Lat. & 59.8 & 83.1 & 44.9 & 73.5 & 63.1 & 83.1 & 28.7 & 42.2 & 38.6 & 60.4 \\
\hline & (38) & (17.0) & $(13.1)$ & (18.5) & $(16.0)$ & (21.9) & (12.3) & $(24.7)$ & (32.8) & (18.3) & $(23.2)$ \\
\hline
\end{tabular}

$\mathrm{P}$ value

$<0.001$

$<0.001$

$<0.001$

$<0.001$

$<0.001$ 
Table 6. Radiographic outcome of different stage of OA stratified by main involved compartment Medial compartment $(\mathrm{N}=611)$ Lateral compartment Total $(\mathrm{N}=649)$ $(\mathrm{N}=38)$

\begin{tabular}{cccccccccc}
\hline Stage & Better & Same & Worse & Rev. DP† & Better & Same & Worse & Rev. DP & Rev. DP \\
& & & & & & & \\
\hline II & 65.2 & 24.2 & 10.6 & $89.4 *$ & 50.0 & 50.0 & 0 & 100 & $89.4 *$ \\
& $(116)$ & $(43)$ & $(19)$ & $(159)$ & $(1)$ & $(1)$ & & $(2)$ & $(161)$ \\
III & 58.6 & 17.8 & 23.6 & 76.4 & 66.7 & 21.2 & 12.1 & 87.9 & 77.3 \\
& $(208)$ & $(63)$ & $(84)$ & $(271)$ & $(22)$ & $(7)$ & $(4)$ & $(29)$ & $(300)$ \\
IV & 52.6 & 23.1 & 24.3 & 75.7 & 66.7 & 33.3 & 0 & 100 & 76.5 \\
& $(41)$ & $(18)$ & $(19)$ & $(59)$ & $(2)$ & $(1)$ & & $(3)$ & $(62)$ \\
\hline Total & 59.7 & 20.3 & 20.0 & 80.0 & 65.8 & 23.7 & 10.5 & 89.5 & 80.1 \\
& $(365)$ & $(124)$ & $(122)$ & $(489)$ & $(25)$ & $(9)$ & $(4)$ & $(34)$ & $(523)$ \\
\hline
\end{tabular}

† Reversed degeneration process $($ Rev. DP) $=$ Better + Same, presented as \% (N)

* Statistically significant by comparisons for each pair using paired t test $(p<0.05)$

Table 7. MRI outcomes stratified by main categories of WORMS

\begin{tabular}{ccccc}
\hline & Cartilage & Bone marrow edema & Bone cyst & Meniscus \\
\hline Get worse & $27.8(10)$ & $36.1(13)$ & $36.1(13)$ & $8.3(3)$ \\
Same & $38.9(14)$ & $41.7(15)$ & $58.3(21)$ & $91.7(33)$ \\
Improved & $33.3(12)$ & $22.2(8)$ & $5.6(2)$ & $0(0)$ \\
\hline †Rev. DP & $72.2(26)$ & $63.9(23)$ & $63.9(23)$ & $91.7(33)$ \\
\hline
\end{tabular}

$\dagger$ Reversed degeneration process $($ Rev. DP) $=$ Better + Same, presented as $\%(N)$

The results of the analyses of factors influencing subjective and radiographic outcomes showed that gender, OA severity (JSW, FTA, X-ray staging, and arthroscopic grading of cartilage damage) are related to subjective outcomes. Age, BMI, pre-operative HA injection, OA severity (JSW, FTA, X-ray staging, arthroscopic grading of cartilage damage) and the type and severity of the medial plica are related to 
radiographic outcomes (Table 8$)$. Thirty knees (4.6\%) of the whole series; twenty-four knees $(6.2 \%)$ in the stage III group and six knees $(7.4 \%)$ in the stage IV group; twenty-nine knees $(4.7 \%)$ in the medial compartment group and one knee (2.6\%) in the lateral compartment group deteriorated and eventually received arthroplasty. The pertinent data for these failed cases are listed in Table 9 . Twenty-four cases $(80 \%)$ of them were arthroscopic grade IV with submeniscus erosion of the tibial plateau and therefore meniscus protrusion. Fifteen (50\%) of them had low compliance with our post-operative self-rehabilitation protocol. Six of these low compliance patients had earlier arthroscopic grades (grade II and III) of main involved compartment and without meniscus protrusion. All of the failed cases with good compliance were arthroscopic grade IV with meniscus protrusion. All of the low compliance cases received TKA; in contrast, only $53.3 \%$ of the cases with good compliance did (the other $46.7 \%$ received UKA). The average elapsed time between ACRFP and arthroplasty was 1.1 years for the group of low compliance compared to 2.6 years for the group of good compliance.

Table 8. Analyses of factors influencing subjective and radiographic outcomes 
Subjective Outcome (Satisfied or not) Radiographic Outcome (RDP or not)

$$
\begin{array}{cccc}
\text { Med. comp. } & \text { Lat. comp. } & \text { Med. comp. } & \text { Lat. comp. } \\
(\mathrm{N}=611) & (\mathrm{N}=38) & (\mathrm{N}=611) & (\mathrm{N}=38)
\end{array}
$$

2x2 Contengency analyses

\begin{tabular}{|c|c|c|c|c|c|}
\hline Sex as female & $\begin{array}{c}0.0134^{*} \\
2.08(1.15-3.77)\end{array}$ & $\begin{array}{c}0.8353 \\
0.78(0.08-8.04)\end{array}$ & $\begin{array}{c}0.3173 \\
1.26(0.80-1.97)\end{array}$ & $\begin{array}{c}0.9478 \\
1.08(0.10-11.92)\end{array}$ & \\
\hline Glucosamine intake & 0.6352 & 0.2489 & 0.4778 & 0.2020 & \\
\hline & $1.05(0.13-8.38)$ & $8(0.41-154.44)$ & $0.39(0.05-3.08)$ & $11(0.54-223.91)$ & \\
\hline HA injection & 0.8625 & 0.6426 & $0.0014 *$ & 0.1509 & \\
\hline & $1.05(0.59-1.86)$ & $0.38(0.04-3.69)$ & $0.51(0.33-0.77)$ & $0.5(0.05-5.03)$ & \\
\hline Previous operation & $\begin{array}{c}0.4144 \\
0.61(0.25-1.51)\end{array}$ & $\begin{array}{c}0.1195 \\
0.15(0.02-1.28)\end{array}$ & $\begin{array}{c}0.8875 \\
1.06(0.50-2.26)\end{array}$ & $\begin{array}{c}1 \\
0.55(0.05-6.25)\end{array}$ & \\
\hline Medial meniscus tear & $\begin{array}{c}0.4116 \\
1.84(0.52-6.47)\end{array}$ & - & $\begin{array}{c}0.5946 \\
0.66(0.19-2.28)\end{array}$ & - & \\
\hline Lateral meniscus tear & $\begin{array}{r}\text { Logis } \\
\text { Data a }\end{array}$ & $\begin{array}{l}0.5272 \\
1.81(0.16-20.55) \\
\text { regression analys } \\
\text { presented as p-va }\end{array}$ & $\begin{array}{l}\text { s } \\
\text { es }\end{array}$ & $\begin{array}{c}0.4456 \\
2.5(0.21-30.22)\end{array}$ & \\
\hline Age & 0.1688 & 0.6635 & $0.0427 *$ & 0.6246 & \\
\hline BMI & 0.2146 & 0.5809 & $<0.0001 *$ & 0.0527 & \\
\hline Duration of symptom & 0.5290 & 0.1025 & 0.3244 & 0.2408 & \\
\hline Pre-op. FTA & $0.0017 *$ & 0.7235 & $<0.0001 *$ & 0.4007 & \\
\hline Pre-op. JSW & $0.0088 *$ & 0.2835 & $0.1089 *$ & 0.9464 & \\
\hline & $\begin{array}{r}\text { Pea } \\
\text { Data a }\end{array}$ & $\begin{array}{l}\text { on's chi-square tes } \\
\text { presented as p-va }\end{array}$ & & & \\
\hline $\begin{array}{c}\text { X-ray staging } \\
\text { Medial }\end{array}$ & $0.0043^{*}$ & 0.6638 & $0.0011^{*}$ & 0.7647 & \\
\hline Lateral & 0.9824 & 0.2391 & 0.6603 & 0.7127 & \\
\hline $\mathrm{PF}$ & 0.8421 & 0.4641 & 0.7295 & 0.2392 & \\
\hline $\begin{array}{c}\text { Medial plica } \\
\text { Type }\end{array}$ & 0.7476 & 0.7307 & $<0.0001^{*}$ & 0.9496 & \\
\hline Severity & 0.6188 & 0.7141 & 0.1043 & $0.0064^{*}$ & \\
\hline $\begin{array}{r}\text { Hidden lesion } \\
\text { Focus A }\end{array}$ & 0.4252 & 0.2153 & 0.0700 & 0.8378 & $\mathrm{RDP}$, \\
\hline Focus B & $0.0011^{*}$ & 0.3391 & 0.0550 & 0.6489 & \\
\hline $\begin{array}{l}\text { Arthroscopic grading } \\
\text { MFC }\end{array}$ & $0.0002 *$ & 0.6234 & $<0.0001 *$ & 0.4501 & \\
\hline MTP & 0.0009* & 0.8870 & $0.0003 *$ & 0.6275 & \\
\hline LC & 0.2112 & 0.7413 & $0.0008 *$ & 0.1366 & \\
\hline PF joint & $0.0418^{*}$ & 0.1517 & $0.0364 *$ & 0.6976 & \\
\hline
\end{tabular}

Data are presented as p-values and odds ratios (95\% confidence interval)

reversed degeneration process; FTA, femorotibial angle; JSW, joint space width; PF, patellofemoral; MFC, medial femoral condyle; MTP, medial tibial plateau; LC, Lateral compartment. * Denotes statistical significance 
Table 9. Pertinent data about the failed cases

\begin{tabular}{|c|c|c|c|c|c|c|c|c|c|}
\hline No. & $\begin{array}{l}\text { Pre- } \\
\text { op. } \\
\text { Stage }\end{array}$ & $\begin{array}{l}\text { Main } \\
\text { Comp. }\end{array}$ & FTA & $\begin{array}{l}\text { JSW } \\
(\mathrm{mm})\end{array}$ & $\begin{array}{c}\text { Arthroscopic } \\
\text { Grading of } \\
\text { Main Comp. }\end{array}$ & $\begin{array}{l}\text { Meniscus } \\
\text { Protrusion }\end{array}$ & $\begin{array}{l}\text { Low Compliance } \\
\text { in Following } \\
\text { Post-op. Protocol }\end{array}$ & $\begin{array}{c}\text { Type of } \\
\text { Arthroplasty }\end{array}$ & $\begin{array}{c}\text { Years } \\
\text { After } \\
\text { ACRFP } \\
\text { When } \\
\text { Failed }\end{array}$ \\
\hline 1 & IV & $\mathrm{M}$ & -6 & 1.10 & IV & $\mathrm{Y}$ & $\mathrm{N}$ & UKA & 3 \\
\hline 2 & III & $\mathrm{M}$ & -2 & 2.62 & IV & $\mathrm{Y}$ & $\mathrm{N}$ & TKA & 4 \\
\hline 3* & IV & $\mathrm{M}$ & -3 & 3.35 & III & $\mathrm{N}$ & $\mathrm{Y}$ & TKA & 1 \\
\hline 4 & IV & $\mathrm{M}$ & -10 & 2.76 & IV & $\mathrm{Y}$ & $\mathrm{N}$ & TKA & 1 \\
\hline 5 & IV & $\mathrm{M}$ & -2 & 1.12 & IV & $\mathrm{Y}$ & $\mathrm{N}$ & UKA & 4 \\
\hline $6^{*}$ & III & $\mathrm{M}$ & 4 & 2.36 & III & $\mathrm{N}$ & $\mathrm{Y}$ & TKA & 2 \\
\hline 7 & III & $\mathrm{M}$ & -5 & 1.01 & IV & $\mathrm{Y}$ & $\mathrm{N}$ & UKA & 3 \\
\hline 8 & III & $\mathrm{L}$ & 8 & 1.22 & IV & $\mathrm{Y}$ & $\mathrm{N}$ & UKA & 3 \\
\hline 9 & III & $\mathrm{M}$ & -2 & 1.35 & IV & $\mathrm{Y}$ & $\mathrm{N}$ & TKA & 1 \\
\hline 10 & III & $\mathrm{M}$ & -1 & 2.59 & IV & $\mathrm{Y}$ & $\mathrm{N}$ & UKA & 2 \\
\hline 11 & III & $\mathrm{M}$ & 0 & 1.92 & IV & $\mathrm{Y}$ & $\mathrm{N}$ & UKA & 2 \\
\hline 12 & III & $\mathrm{M}$ & -3 & 2.40 & IV & $\mathrm{Y}$ & $\mathrm{N}$ & UKA & 1 \\
\hline 13 & III & $\mathrm{M}$ & -5 & 1.12 & IV & $\mathrm{Y}$ & $\mathrm{Y}$ & TKA & 1 \\
\hline 14 & IV & $\mathrm{M}$ & -10 & 0.00 & IV & $\mathrm{Y}$ & $\mathrm{Y}$ & TKA & 1 \\
\hline 15 & IV & $\mathrm{M}$ & -11 & 0.00 & IV & $\mathrm{Y}$ & $\mathrm{N}$ & TKA & 3 \\
\hline 16 & III & $\mathrm{M}$ & -3 & 2.20 & IV & $\mathrm{Y}$ & $\mathrm{Y}$ & TKA & 1 \\
\hline 17 & III & $\mathrm{M}$ & -3 & 2.46 & IV & $\mathrm{Y}$ & $\mathrm{Y}$ & TKA & 1 \\
\hline 18 & IV & $\mathrm{M}$ & -5 & 0.86 & IV & $\mathrm{Y}$ & $\mathrm{Y}$ & TKA & 1 \\
\hline 19 & IV & $\mathrm{M}$ & -4 & 1.78 & IV & $\mathrm{Y}$ & $\mathrm{Y}$ & TKA & 1 \\
\hline $20 *$ & III & $\mathrm{M}$ & -3 & 2.54 & III & $\mathrm{N}$ & $\mathrm{Y}$ & TKA & 1 \\
\hline 21 & III & $\mathrm{M}$ & -4 & 1.96 & IV & $\mathrm{Y}$ & $\mathrm{N}$ & TKA & 4 \\
\hline 22 & IV & $\mathrm{M}$ & -5 & 1.01 & IV & $\mathrm{Y}$ & $\mathrm{N}$ & TKA & 4 \\
\hline 23 & IV & $\mathrm{M}$ & -5 & 0.66 & IV & $\mathrm{Y}$ & $\mathrm{Y}$ & TKA & 1 \\
\hline $24 *$ & III & $\mathrm{M}$ & 2 & 3.88 & II & $\mathrm{N}$ & $\mathrm{Y}$ & TKA & 1 \\
\hline $25 *$ & III & $\mathrm{M}$ & 1 & 3.20 & II & $\mathrm{N}$ & $\mathrm{Y}$ & TKA & 1 \\
\hline 26 & IV & $\mathrm{M}$ & -5 & 0.98 & IV & $\mathrm{Y}$ & $\mathrm{Y}$ & TKA & 1 \\
\hline $27 *$ & III & $\mathrm{M}$ & 0 & 2.34 & III & $\mathrm{N}$ & $\mathrm{Y}$ & TKA & 1 \\
\hline 28 & III & $\mathrm{M}$ & -4 & 1.44 & IV & $\mathrm{Y}$ & $\mathrm{Y}$ & TKA & 2 \\
\hline 29 & IV & $\mathrm{M}$ & -10 & 0.00 & IV & $\mathrm{Y}$ & $\mathrm{N}$ & TKA & 1 \\
\hline 30 & IV & $\mathrm{M}$ & -3 & 1.56 & IV & $\mathrm{Y}$ & $\mathrm{N}$ & TKA & 3 \\
\hline
\end{tabular}


Pre-op., pre-operative; Comp., compartment; FTA, femorotibial angle; JSW, joint space width; Post-op., postoperative; TKA, total knee arthroplasty; UKA, unicompartmental knee arthroplasty

* Denotes cases with earlier arthroscopic grading of II or III and without meniscus protrusion

\section{Evidence of Cartilage Regeneration}

The radiographic images as demonstrated in Figures 3 and 4 showed indirect evidence of cartilage regeneration. Furthermore, the MRI studies also disclosed the possibility of cartilage regeneration as shown in Fig 5.

\section{Discussion}

In this report we present the surgical procedure, the standard post-operative self-rehabilitation protocol and the clinical and radiographic outcomes of ACRFP for knee OA. Overall, the subjective satisfactory rate was $91.1 \%$. Even in stage IV cases, $91.4 \%$ of the patients were satisfied after at least 3 years of follow-up. The KSS and KOOS surveys showed improvements of clinical symptoms and quality of life, and the radiographic evaluations and comparisons showed that the revitalization of the degenerated cartilage and the reversal of the degeneration process were observed in $80.1 \%$ of the knees after the ACRFP. The oneyear MRI outcome study also demonstrated reversal of the degeneration process of cartilage in $72.2 \%$ of the randomly selected stage III cases.

The mediopatellar plica is a fold in the synovium that represents an embryologic remnant in the development of the knee's synovial cavity [20], [21]. Although the prevalence of medial plica ranges widely, from $18 \%$ to $95 \%$ [22]-[24], it is generally agreed that this structure can produce knee pain and can be successfully treated by arthroscopic resection in the event that it becomes inflamed, thickened, and/or less elastic [22], [25], [26]. Abrasion phenomenon between the medial plica and the opposite medial femoral condyle has been described in patients with knee OA [6], [27], [28]. The repeated injuries elicited by this abrasion phenomenon was found to trigger IL-1ß production, thus enhance the expression of MMP-3. The expression of IL1-ß mRNA, MMP-3 mRNA and MMP-3 in the medial plica of the knees with early stage knee OA suggests that medial plica and its interplay with its facing medial femoral condyle might play an important role in the pathogenesis of medial compartment OA knee [10]. A three-dimensional dynamic finate element model has demonstrated that the close relationship and possible high contact pressure between a fibrotic medial plica and medial femoral condyle during knee motion might be a cause of cartilage damage on the medial femoral condyle [12]. These findings support the fact that using arthroscopic release to eradicate fibrotic medial plica could be effective in symptom relief, and it might even modify the disease process of OA knees [14], [15].

The subjective satisfactory rate of $91.1 \%$ in this study is better than the previous report [15] of $85.5 \%$ ( $91.4 \%$ vs. $59 \%$ in stage IV cases). This might be the consequence of technique improvement by 
standardizing the surgical procedure and more skillful in conducting post-operative self-rehabilitation program under the monitoring of case managers. In this study, the analysis of the factors influencing outcomes revealed that gender and OA severity were related to subjective outcomes. Age, BMI, preoperative $\mathrm{HA}$ injection, $\mathrm{OA}$ severity and the type and severity of the medial plica are important predictors of radiographic outcomes. The negative correlation of age, BMI and severity of OA with outcomes is compatible with common understanding and points to the importance of early ACRFP intervention. The finding of female gender having better subjective outcomes might be explained by their hypersensitivity to pain [29]. Patients who had received preoperative HA injection had worse radiographic outcome; this might be due to reactive arthrofibrosis or infrapatellar fat pad fibrosis which are common arthroscopic findings in patients who have received multiple injections or ever experienced failed extraarticular injections. The masking effect of HA injections might also have delayed the ACRFP intervention. The analysis of failed cases reconfirmed that early intervention and skillful post-operative care both help make ACRFP successful. Patients having low compliance with our self-rehabilitation protocol thus developing arthrofibrosis are more likely to receive earlier or even unnecessary TKA (Cases 3, 6, 20, 24, 25, and 27 in Table 8).

Although articular hyaline cartilage was classically considered as having no or low potential for regeneration [30], both direct and indirect evidence of articular cartilage regeneration have been reported after correction of varus deformity for knee OA by some authors [31], [32]. Previous reports on arthroscopic medial release and ACRFP [14], [15] also demonstrated that by removing all existing catabolic factors, the anabolism of the damaged cartilage might become dominant and regeneration ensues. The radiographic observation of the reversal of the degeneration process in $80.1 \%$ of the knees in this study coincides with the findings of previous report of $81.2 \%$ [15]. In this report, the one-year MRI outcome study further demonstrated reversal of the degeneration process of cartilage in $72.2 \%$ of the randomly selected stage III cases.

Mechanical stresses are known to play an important role on articular cartilage degradation [33]. It has been shown that static compression suppresses matrix biosynthesis, while cyclic and intermittent loading can either stimulate or suppress matrix synthesis, depending on the frequency or magnitude of loading. High rates or magnitudes of stress can induce an "injurious" response that has been associated with increased degradation, cell death, and the production of matrix metalloproteinases [34]. Technologies such as wedge insoles, unloading knee brace, high tibial osteotomy, and knee joint distraction that "unload" the joint may reverse the structural damage [35]. Regarding the anabolic effects of ACRFP, in addition to the elimination of the physical abrasion damage of MAP [12] and the chemical erosion of focal inflammatory tissues [10], another important factor is the releasing of the increased static pressure in the patellofemoral joint which is caused by lifelong, repeated inflammation induced by MAP. The beneficial effect of modification of dynamic foot pressure and gait pattern after ACRFP was also observed by a recent gait analysis study [36].

In contrast to the uncertain beneficial mechanism and the diverse outcomes of common arthroscopic techniques including lavage, debridement, abrasion arthroplasty, microfracture, and autologous

Page $14 / 23$ 
chondrocyte implantation (ACl) for knee OA [37]-[41], the concept of ACRFP has more precise rationale of treatment and more reproducible, beneficial outcomes. ACRFP was developed and conceptualized according to a series of studies [6]-[12] regarding MAP as a cause of knee OA [13]. The outcomes of this study affirms that, besides eradicating the MAP, the key to a successful ACRFP is to adequately release the overtightness of the patellofemoral joint and maintain this appropriate tension around patella with skillful supervised post-operative self-rehabilitation to prevent recurrent tightness due to scarring and arthrofibrosis.

However, there are limitations in this study. In order to get more convincing evidence, properly conducted randomized control clinical trials and massive long-term MRI evaluations are warranted.

\section{Conclusion}

The findings of cartilage regeneration after ACRFP in this study substantiate the hypothesis that MAP is an important factor in the pathogenesis of knee OA. Moreover, if performed in time, the ACRFP combined with skillful post-operative self-rehabilitation protocol has demonstrated that re-establishing a favorable biomechanical environment for knees may satisfy the majority of patients with knee $\mathrm{OA}$ and delay the need for invasive joint reconstruction procedures.

\section{Abbreviations}

$\mathrm{OA}=$ osteoarthritis

ACRFP $=$ arthroscopic cartilage regeneration facilitating procedure

KSS $=$ knee society score

KOOS = knee injury and osteoarthritis outcome score

$\mathrm{MRI}=$ magnetic resonance imaging

$\mathrm{BMI}=$ body mass index

MAP = medial abrasion phenomenon

$\mathrm{KHPO}=$ knee health promotion option

$\mathrm{HA}=$ hyaluronic acid

FSE $=$ fast spin echo

$F S=$ fat saturation

$\mathrm{TR} / \mathrm{TE}=$ repitition time/echo time 
NEX = number of excitation

PACS = picture archiving communication system

FTA = femorotibial angle

MJSW = minimal joint space width

WORMS = whole organ MRI score

ANOVA = analysis of variance

UKA = unicompartmental knee arthroplasty

TKA = total knee arthroplasty

$\mathrm{ACl}=$ autologous chondrocyte implantation

\section{Declarations}

\section{Ethics approval and consent to participate}

This study was approved by the Research Ethics Committee of Dalin Tzu-Chi General Hospital (Approval Number: B09704022). Written informed consent was obtained from all the participants.

\section{Consent for publication}

Written informed consent was obtained from the patients for publication of this research and any accompanying images.

\section{Availability of data and materials}

The datasets during and/or analysed during the current study are available from the corresponding author on reasonable request.

\section{Competing interests}

The authors declare that they have no competing interests.

\section{Funding}

No funding was received.

\section{Authors' contributions}

SRL and CYR were involved in the design of the study and performed the statistical analysis. SRL and $\mathrm{CCH}$ performed the operations. CWL and CYR evaluated the radiographic outcomes. SRL, JPH and CYR 
collected data. SRL and CYR were responsible for drafting the paper and revising it. All authors have read and approved the final manuscript.

\section{Acknowledgements}

None.

\section{References}

[1] L. March, M. Cross, N. Arden, and G. Hawker, "Osteoarthritis: A serious disease," OARSI, 2016.

[2] D. Ruiz et al., "The direct and indirect costs to society of treatment for end-stage knee osteoarthritis," J. Bone Jt. Surg. - Ser. A, 2013.

[3] N. Hussain, H. Johal, and M. Bhandari, "An evidence-based evaluation on the use of platelet rich plasma in orthopedics - a review of the literature," SICOT-J, 2017.

[4] R. Bastos et al., "Intra-articular injections of expanded mesenchymal stem cells with and without addition of platelet-rich plasma are safe and effective for knee osteoarthritis," Knee Surgery, Sport. Traumatol. Arthrosc., 2018.

[5] A. A. Balaji Saibaba, "Emergence of Orthobiologics as a Novel Therapeutic Modality for Osteoarthritis of Knee," Rheumatol. Curr. Res., 2015.

[6] S.-R. Lyu and C.-C. Hsu, "Medial plicae and degeneration of the medial femoral condyle," Arthrosc. J. Arthrosc. Relat. Surg., vol. 22, no. 1, 2006.

[7] S.-R. Lyu, J.-E. Tzeng, C.-Y. Kuo, A.-R. Jian, and D.-S. Liu, "Mechanical strength of mediopatellar plica - The influence of its fiber content," Clin. Biomech., vol. 21, no. 8, 2006.

[8] S.-R. Lyu, "Relationship of medial plica and medial femoral condyle during flexion," Clin. Biomech., vol. 22, no. 9, 2007.

[9] S.-R. Lyu, J.-K. Chiang, and C.-E. Tseng, "Medial plica in patients with knee osteoarthritis: A histomorphological study," Knee Surgery, Sport. Traumatol. Arthrosc., vol. 18, no. 6, 2010.

[10] H.-S. Wang, P.-Y. Kuo, C.-C. Yang, and S.-R. Lyu, "Matrix metalloprotease-3 expression in the medial plica and pannus-like tissue in knees from patients with medial compartment osteoarthritis," Histopathology, vol. 58, no. 4, 2011.

[11] C.-C. Yang, C.-Y. Lin, H.-S. Wang, and S.-R. Lyu, "Matrix metalloproteases and tissue inhibitors of metalloproteinases in medial plica and pannus-like tissue contribute to knee osteoarthritis progression," PLoS One, vol. 8, no. 11, 2013. 
[12] D. S. Liu, Z. W. Zhuang, and S. R. Lyu, "Relationship between medial plica and medial femoral condyle - A three-dimensional dynamic finite element model," Clin. Biomech., vol. 28, no. 9-10, 2013.

[13] S.-R. Lyu et al., "Role of medial abrasion phenomenon in the pathogenesis of knee osteoarthritis," Med. Hypotheses, vol. 85, no. 2, 2015.

[14] S.-R. Lyu, "Arthroscopic medial release for medial compartment osteoarthritis of the knee: the result of a single surgeon series with a minimum follow-up of four years.," J. Bone Joint Surg. Br., vol. 90, no. 9, pp. 1186-1192, Sep. 2008.

[15] S.-R. Lyu, C.-C. Hsu, and C.-W. Lin, "Arthroscopic cartilage regeneration facilitating procedure for osteoarthritic knee.," BMC Musculoskelet. Disord., vol. 13, no. 1, p. 226, 2012.

[16] S.-R. Lyu, "Knee health promotion option for knee osteoarthritis: A preliminary report of a concept of multidisciplinary management," Heal. Aging Res., 2015.

[17] C. Slattery and C. Y. Kweon, "Classifications in Brief: Outerbridge Classification of Chondral Lesions," Clin. Orthop. Relat. Res., 2018.

[18] G. B. Joseph et al., "Texture analysis of cartilage T2 maps: Individuals with risk factors for OA have higher and more heterogeneous knee cartilage MR T2 compared to normal controls - data from the osteoarthritis initiative," Arthritis Res. Ther., 2011.

[19] C. G. Peterfy et al., "Whole-organ magnetic resonance imaging score (WORMS) of the knee in osteoarthritis," Osteoarthr. Cartil., 2004.

[20] S. Ogata and H. K. Uhthoff, "The development of synovial plicae in human knee joints: An embryologic study," Arthrosc. J. Arthrosc. Relat. Surg., 1990.

[21] J. Y. Dupont, "Synovial plicae of the knee: Controversies and review," Clin. Sports Med., 1997.

[22] H. Zmerly, I. Akkawi, R. Citarella, and M. E. Ghoch, "Clinical Management of Medial Patellar Plica Syndrome: Mini Review from Diagnosis to Treatment," Curr. Rheumatol. Rev., 2018.

[23] A. Nakayama, T. Sugita, T. Aizawa, A. Takahashi, and T. Honma, "Incidence of medial plica in 3,889 knee joints in the Japanese population," Arthrosc. - J. Arthrosc. Relat. Surg., 2011.

[24] M. R. Gurbuz H, Calpur OU, Ozcan M, Kutoglu T, "The synovial plicae in the knee joint.," Saudi Med J., vol. Dec;27, no. 12, pp. 1839-42, 2006.

[25] S. S. Bellary et al., "Medial plica syndrome: A review of the literature," Clinical Anatomy. 2012.

[26] O. S. Schindler, "'The Sneaky Plica' revisited: Morphology, pathophysiology and treatment of synovial plicae of the knee," Knee Surgery, Sport. Traumatol. Arthrosc., 2014. 
[27] D. Hayashi et al., "Prevalence of MRI-detected mediopatellar plica in subjects with knee pain and the association with MRI-detected patellofemoral cartilage damage and bone marrow lesions: Data from the Joints on Glucosamine study," BMC Musculoskelet. Disord., 2013.

[28] S.-R. Lyu, C.-C. Lee, and C.-C. Hsu, "Medial abrasion syndrome: A neglected cause of knee pain in middle and old age," Med. (United States), vol. 94, no. 16, 2015.

[29] E. J. Bartley and R. B. Fillingim, "Sex differences in pain: A brief review of clinical and experimental findings," Br. J. Anaesth., 2013.

[30] J. C. Bernhard and G. Vunjak-Novakovic, "Should we use cells, biomaterials, or tissue engineering for cartilage regeneration?," Stem Cell Research and Therapy. 2016.

[31] T. Koshino, S. Wada, Y. Ara, and T. Saito, "Regeneration of degenerated articular cartilage after high tibial valgus osteotomy for medial compartmental osteoarthritis of the knee," Knee, 2003.

[32] T. Kanamiya, M. Naito, M. Hara, and I. Yoshimura, "The influences of biomechanical factors on cartilage regeneration after high tibial osteotomy for knees with medial compartment osteoarthritis: Clinical and arthroscopic observations," Arthroscopy, 2002.

[33] T. Davisson, S. Kunig, A. Chen, R. Sah, and A. Ratcliffe, "Static and dynamic compression modulate matrix metabolism in tissue engineered cartilage," J. Orthop. Res., 2002.

[34] G. Sharma, R. K. Saxena, and P. Mishra, "Differential effects of cyclic and static pressure on biochemical and morphological properties of chondrocytes from articular cartilage," Clin. Biomech., 2007.

[35] C. Waller, D. Hayes, J. E. Block, and N. J. London, "Unload it: The key to the treatment of knee osteoarthritis," Knee Surgery, Sport. Traumatol. Arthrosc., 2011.

[36] T. C. Wu, C. H. Yen, S. R. Lyu, and S. S. Hung, "MODIFICATION IN FOOT PRESSURE AND GAIT PATTERN AFTER ARTHROSCOPIC CARTILAGE REGENERATION FACILITATING PROCEDURES (ACRFP) IN PATIENTS WITH OSTEOARTHRITIS OF KNEE," in Journal of Mechanics in Medicine and Biology, 2019.

[37] W. Laupattarakasem, M. Laopaiboon, P. Laupattarakasem, and C. Sumananont, "Arthroscopic debridement for knee osteoarthritis," Cochrane Database of Systematic Reviews. 2008.

[38] M. Pitta, W. Davis, and E. H. Argintar, "Arthroscopic management of osteoarthritis," Journal of the American Academy of Orthopaedic Surgeons. 2016.

[39] K. Mithoefer, T. Mcadams, R. J. Williams, P. C. Kreuz, and B. R. Mandelbaum, "Clinical efficacy of the microfracture technique for articular cartilage repair in the knee: An evidence-based systematic analysis," American Journal of Sports Medicine. 2009. 
[40] J. B. Thorlund, C. B. Juhl, E. M. Roos, and L. S. Lohmander, "Arthroscopic surgery for degenerative knee: Systematic review and meta-analysis of benefits and harms," British Journal of Sports Medicine. 2015.

[41] J. D. Marsh et al., "Cost-effectiveness analysis of arthroscopic surgery compared with non-operative management for osteoarthritis of the knee," BMJ Open, 2016.

\section{Figures}

a

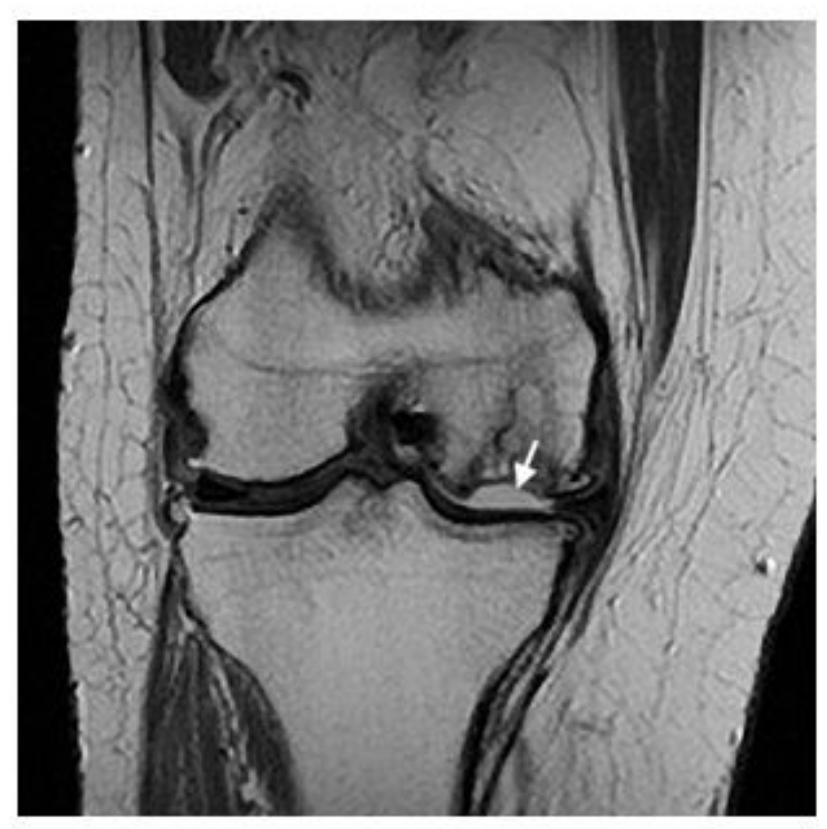

b

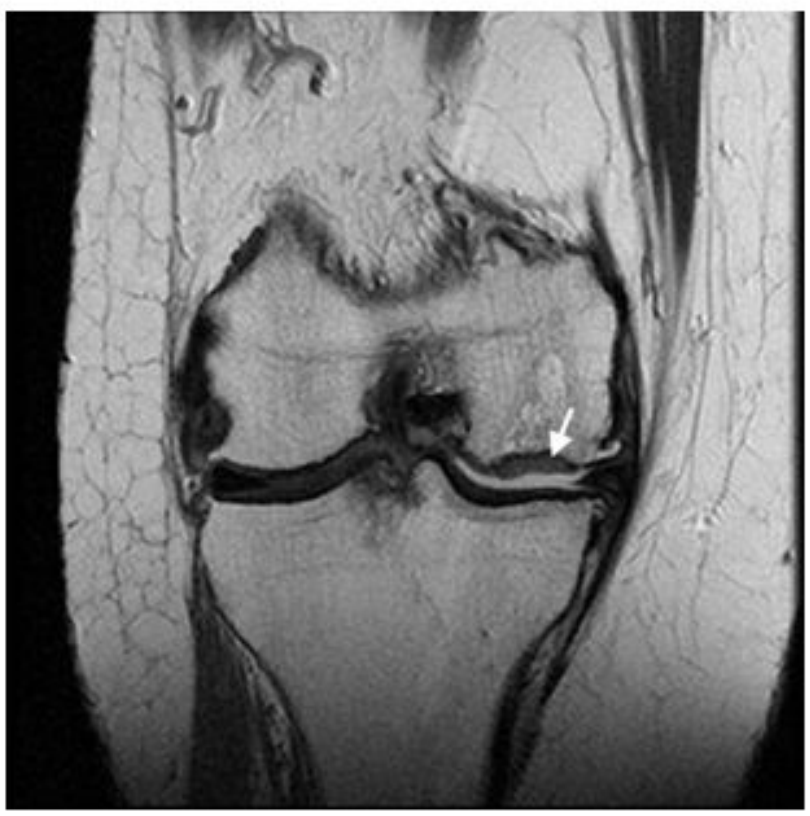

\section{Figure 1}

MRI evidence of cartilage regeneration. a, preoperative MR image of a 68-year-old female with grade III OA over medial compartment of her right knee, diffuse full thickness loss of cartilage (arrow) was found over medial condyle of her right knee; $b$, one year follow-up image showed apparent regeneration of cartilage (arrow). 

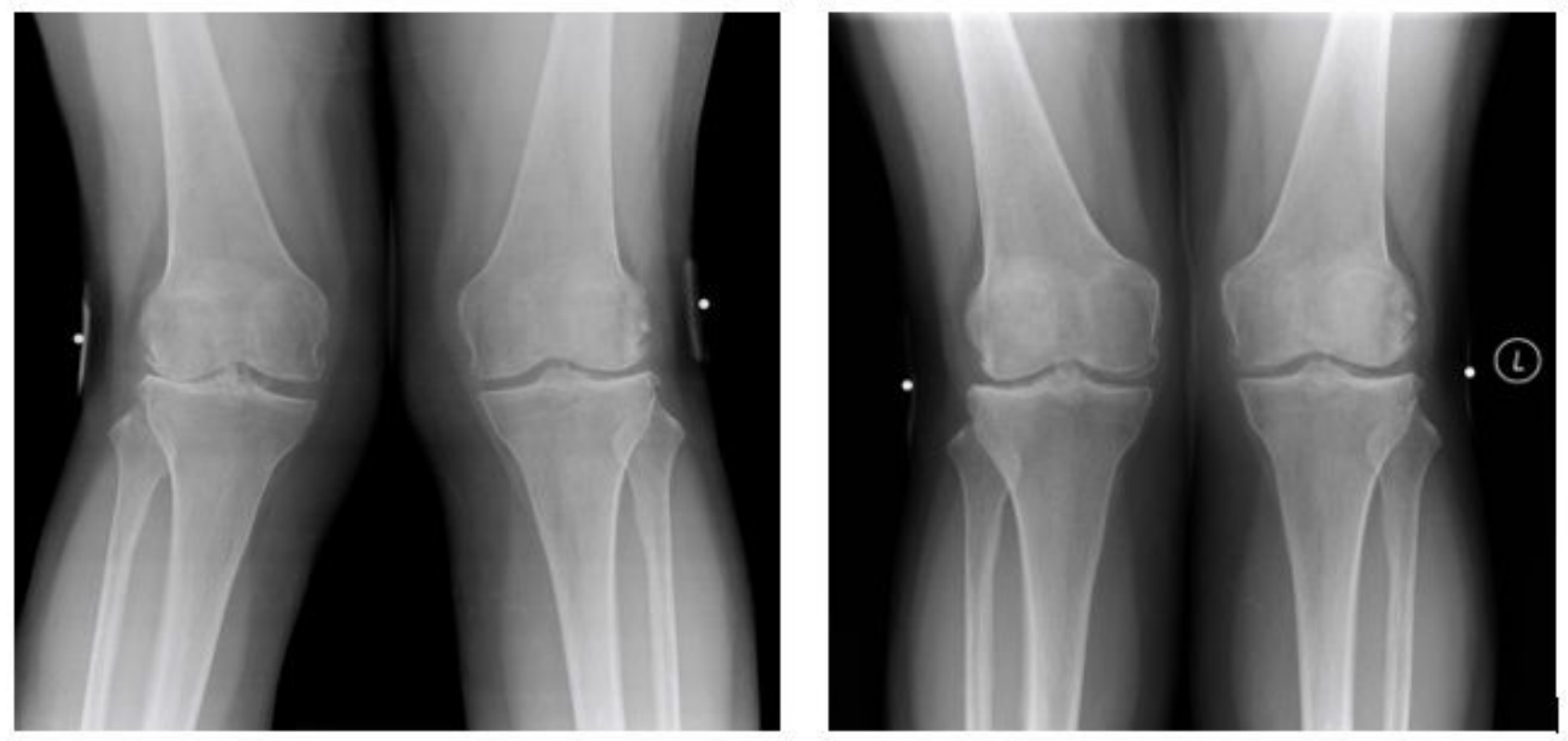

\section{Figure 2}

Another example of the reversal of the degeneration process after receiving ACRFP. a, pre-operative AP standing view of a 67-year-old female with grade IV OA over lateral compartment of her right knee and grade III OA over lateral compartment of her left knee, ACRFP was performed for both knees; b, 34 months later, noticeable improvement of the radiographic manifestation could be observed for both of her knees.

a

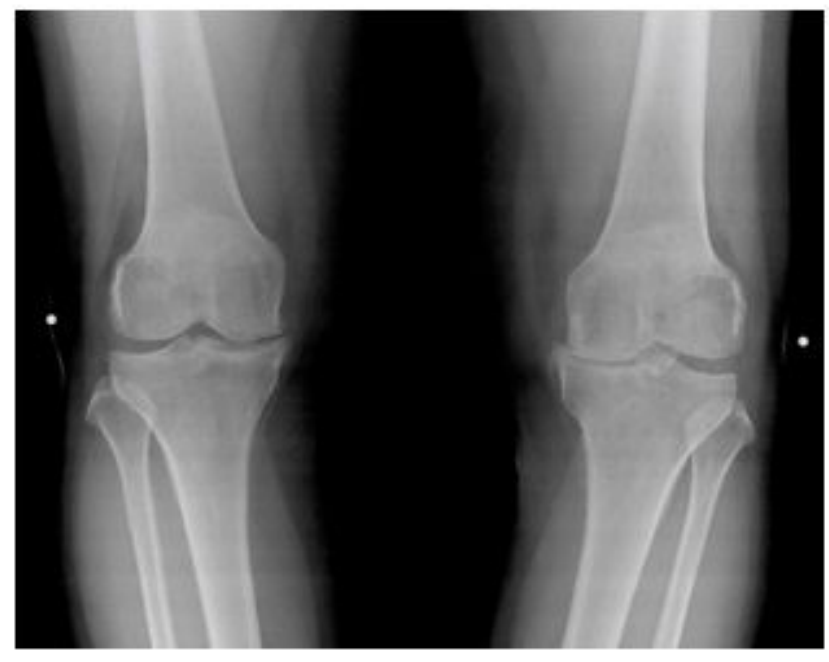

b

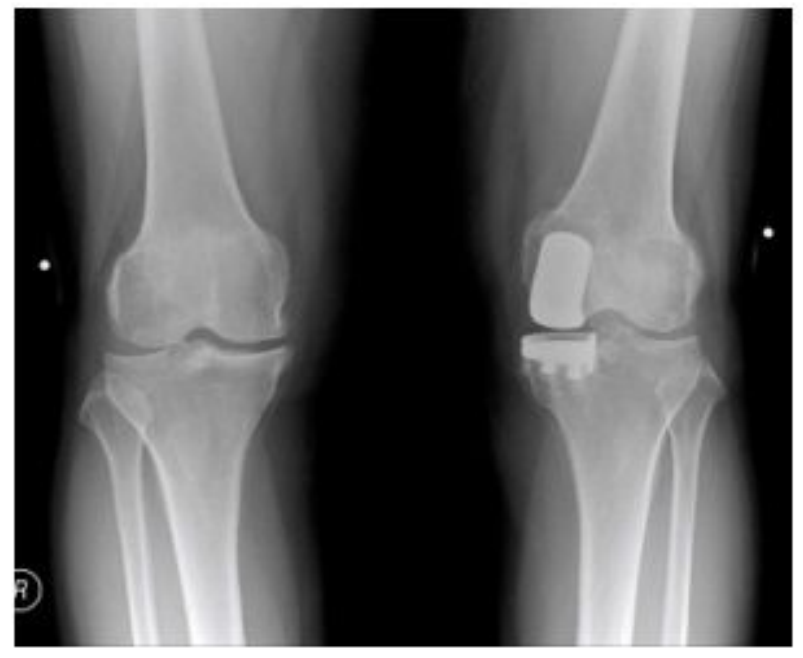

\section{Figure 3}

An example of the reversal of the degeneration process after receiving ACRFP. a, AP standing view of a 69year-old female patient having grade $\mathrm{V}$ OA over medial compartment of her left knee and grade III OA over 
medial compartment of her right knee, unicompartmental arthroplasty for her left knee and ACRFP for her right knee was recommended; b, 39 months later, obvious improvement of the radiographic manifestation could be observed for both of her knees.

a

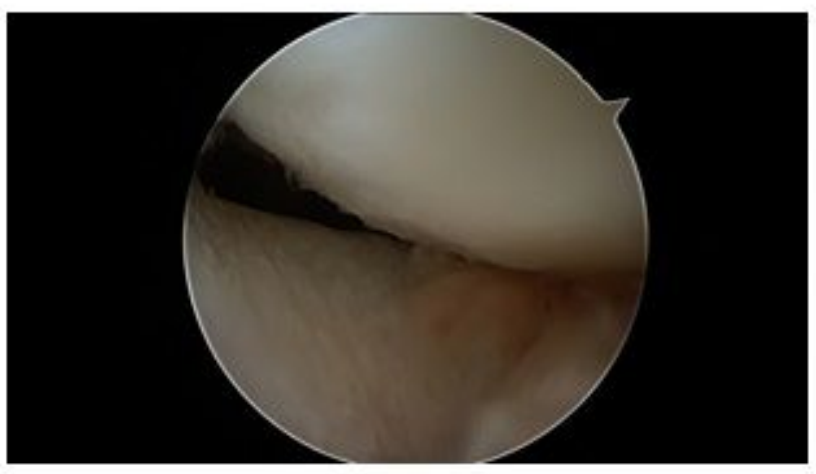

C

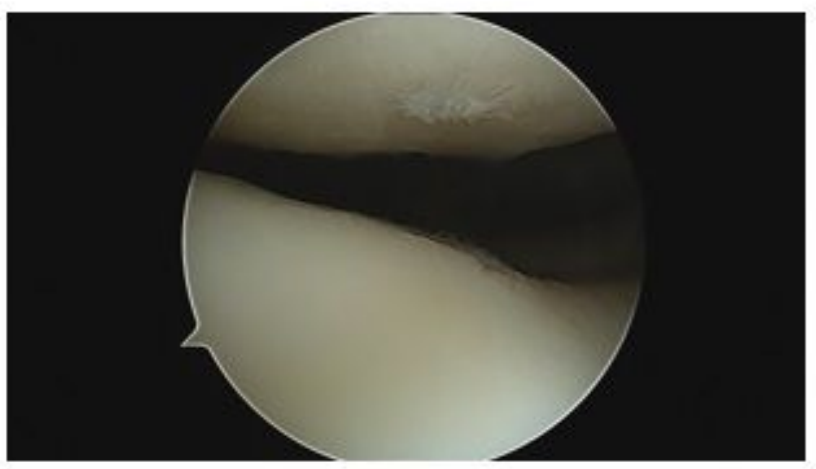

b

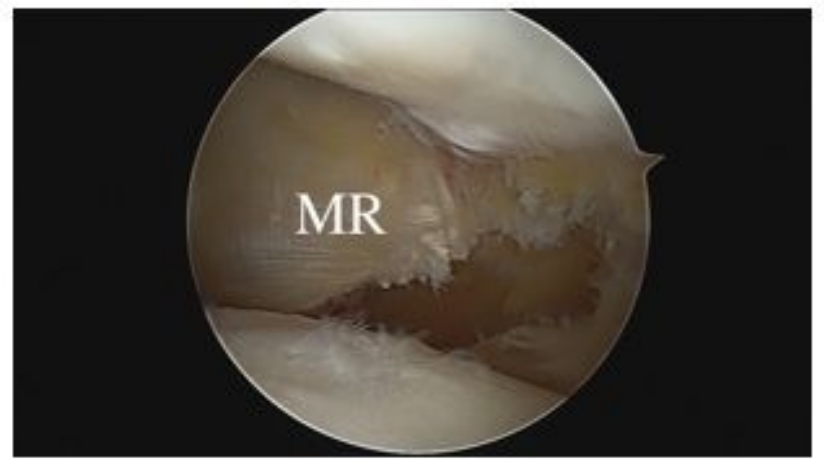

$\mathrm{d}$

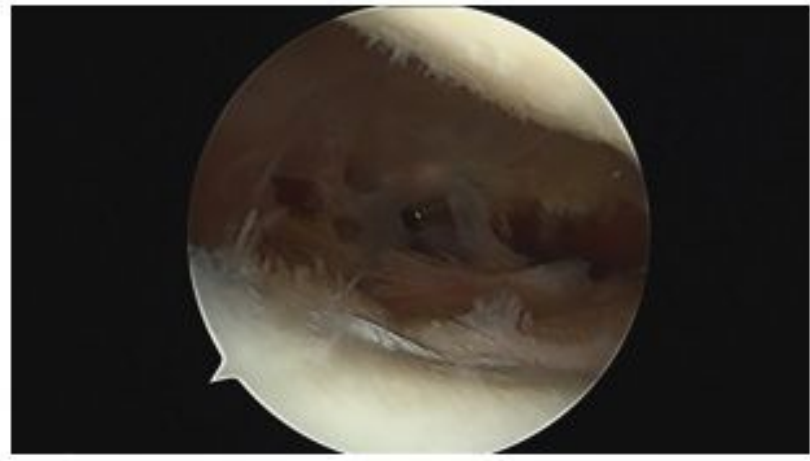

\section{Figure 4}

The decompressing effects of ACRFP. a, after adequate medial release, the previously tight medial patellofemoral joint space was opened; $b$, the medial retinaculum (MR) was visualized; $c$, after medial release, the patella would always deviate laterally; $d$, after percutaneous lateral release, the lateral patellofemoral joint was wide open. 
a

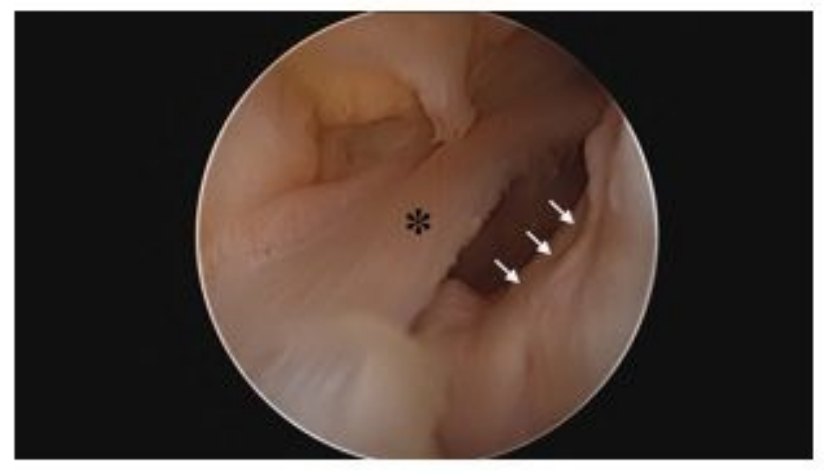

C

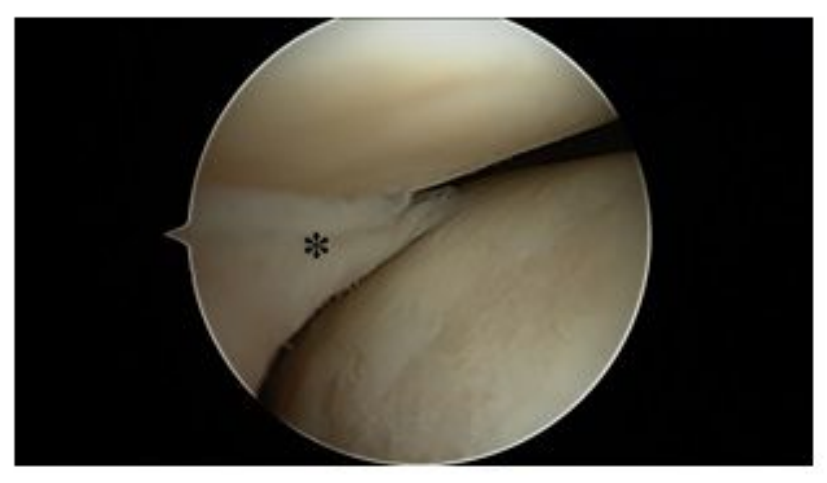

b

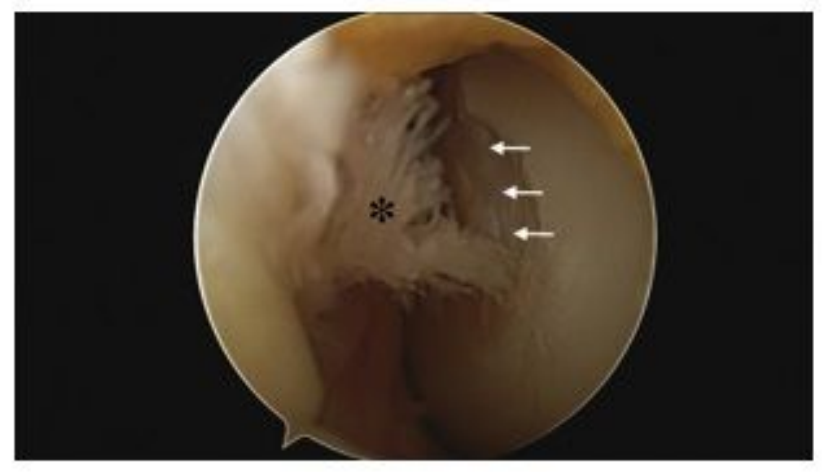

d

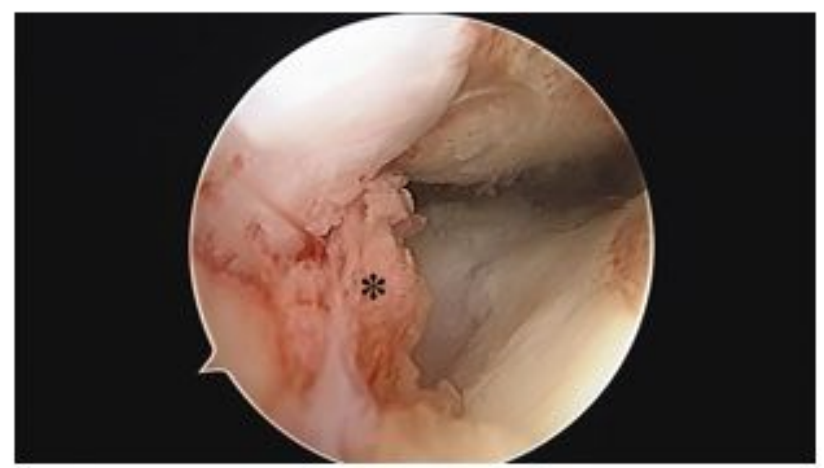

\section{Figure 5}

Medial abrasion phenomenon and its sequelae. a, pathologic medial plica $\left(^{*}\right)$ and lesion "A" (arrows) on the medial femoral condyle caused by focal abrasion; $b$, lesion "B" (arrows) on the medial femoral condyle caused by synovitis on the medial plica $\left(^{*}\right)$; c, impingement of medial plica $\left(^{*}\right)$ and tightness of patellofemoral joint due to chronic inflammation; $d$, focal synovitis $\left(^{*}\right)$ over the inferomedial region of the patella.

\section{Supplementary Files}

This is a list of supplementary files associated with this preprint. Click to download.

- ACRFP.mp4 